\title{
Minimally Invasive Pedicle Subtraction Osteotomy via Tubular Retractor for Adult Rigid Spinal Kyphoscoliosis: a Case Report and Review of the Literature
}

\author{
ZeJun Xing \\ Shanxi Bethune Hospital https://orcid.org/0000-0001-9149-5229 \\ Zhuo Ma \\ Shanxi Bethune Hospital \\ Xun Ma ( $\nabla$ 18735130965@163.com ) \\ Shanxi Bethune Hospital
}

\section{Technical note}

Keywords: adult rigid spinal kyphoscoliosis, minimally invasive, pedicle subtraction osteotomy

Posted Date: January 5th, 2021

DOI: https://doi.org/10.21203/rs.3.rs-138937/v1

License: (c) (1) This work is licensed under a Creative Commons Attribution 4.0 International License.

Read Full License 


\section{Abstract}

Objective To describe a new technique, minimally invasive pedicle subtraction osteotomy (MIS PSO) via tubular retractor, for severe rigid adult spinal deformity.

Methods We present a case with severe rigid thoracolumbar kyphoscoliosis and describe the surgical procedure, to which this patient responded favorably.

Results No surgical complications were seen and estimated blood loss was $600 \mathrm{ml}$. The operation process time was about 5 hours. She received 2 unit of packed red blood cells intraoperatively. VAS was 8.5 points at pre-operation and 2.0 points at 2 years follow-up. The ODI score improved from 33 to 9. Fusion was confirmed by wedging of L2 vertebral body on CT reconstructive films. Routine x-rays showed that spinal imbalance was corrected and maintained at the final follow up. Maximal ambulation distance increased to 5000 feet at 2 years follow-up.

Conclusion A MIS PSO is a feasible option for the treatment of certain subtypes of adult spinal deformity. It is technically feasible that although limited by learning curve of minimally invasive tubular technique and open PSO technique. Both operation skill of the surgeons and proper patient selection is the key to successfully treating adult patients with deformity using MIS PSO.

\section{Introduction}

Adult spinal deformity (ASD) is a common disorder with an increasing clinical and societal impact on the elderly population all over the world. Some reports have noted the prevalence of ASD to be as high as $60 \%$ in the older population. [1] Such deformities can involve any combination of the axial, coronal and sagittal planes, which correlates with poor clinical outcomes. [2]

To restore sagittal balance and make the patient stand erect is the primary goal of the surgical treatment of patients with fixed sagittal imbalance. [3,4] In 1985, Thomasen[5] first described the three-column posterior wedge osteotomy for the management of fixed sagittal deformities in patients with ankylosing spondylitis. Thereafter, pedicle subtraction osteotomy (PSO) has been commonly used for the correction of fixed sagittal imbalance. A PSO is typically used in patients with greater imbalances in the sagittal plane of the spine and when a minimum of 30 degrees of correction is needed. [6, 7] However, a PSO is a technically demanding procedure associated with substantial surgical bleeding and notably high complication rates. [3, 8]Buchowski et al. reported the prevalence of intra-operative and post-operative neurological deficits to be $11.1 \%$ and the prevalence of permanent deficits to be $2.8 \%$ in a study of 108 patients who had undergone a PSO.[9] Furthermore, the main problems of open surgery are often limited by the patient's age, medical comorbidities, as well as the considerable blood loss expected during open surgery.

Minimally invasive surgery (MIS) minimizes injuries to the paraspinal muscles through the use of specialized instrumentation and surgical techniques. MIS has become a fundamental part of spinal 
surgical practice due to the promise of decreased morbidity and faster recovery time. Furthermore, there has been an increased demand from patients seeking MIS treatment for their spinal diseases. $[10,11,12]$ To our knowledge, minimally invasive pedicle subtraction osteotomy (MIS PSO) via tubular retractor for the treatment of adult rigid spinal kyphoscoliosis has not been reported. Here we describe a case of adult rigid spinal kyphoscoliosis with MIS PSO via tubular retractor.

\section{Case Report}

\section{The patient history and clinical evaluation}

The patient was a 74-year-old woman who lived in the rural areas of North China. She was thin and $154 \mathrm{~cm}$ in height. She complained that she could not lie flat on a bed, with an aching back while trying to stand erect or walk for a long time. She endured this chronic illness and fell into despondency due to failure of long-term conservative treatment.

Physical examination showed that she had severe kyphosis and limited motion of the spine. The strength and sensation of the lower limbs were normal, and the physiological reflex was present. Her physical condition was acceptable, and no obvious contraindication was found.

The clinical outcome was evaluated using the Oswestry Disability Index (ODI) and back pain evaluated by the Visual Analog Scale method (VAS score): 0 represented no pain and 10 reflected the most unbearable pain preoperatively and at finial follow-up. Maximal ambulation distance without pain was also recorded.

\section{Radiological evaluation}

Standing 36-inch posterior-anterior (PA) and lateral views were used to evaluate global spinal balance and pelvic parameters before and after surgery. This included coronal balance (C7-CSVL, horizontal distance between a line drawn vertically through the center of the sacrum (CSVL), and a plumb line dropped from the $\mathrm{C} 7$ centroid), coronal Cobb angle (the coronal cobb angle from the upper-end vertebra to lower-end vertebra ), thoracic kyphosis (TK, the sagittal cobb angle from the upper endplate of T5 to the lower endplate of T12), lumbar lordosis (LL, the sagittal cobb angle from the upper endplate of L1 to the upper endplate of S1), and global kyphosis (GK, the sagittal cobb angle from the upper endplate of upper end vertebra to the lower endplate of lower end vertebra), sacral slope (SS, the angle between the horizontal and the sacral plate), pelvic tilt (PT, the angle between the vertical and the line through the midpoint of the sacral plate to femoral heads axis), pelvic incidence ( $\mathrm{PI}$, the angle subtended by a perpendicular from the upper endplate of $\mathrm{S} 1$ and a line connecting the center of the femoral head to the center of the upper endplate of S1), sagittal vertical axis (SVA, the horizontal offset from the posteriorsuperior corner of $\mathrm{S} 1$ to the vertebral body of $\mathrm{C7}$ ). Sagittal and coronal imbalance was defined as an imbalance greater than $5 \mathrm{~cm}$. By convention, a C7PL that falls anterior or posterior to the posterior superior sacrum is designated as positive or negative, respectively. 
Additional radiographs that provided important information to evaluate spinal deformity included standing dynamic radiographs and supine side-bending radiographs (Fig. 1). Computed tomography (CT) images were also obtained to verify deformity structures pre-operation and to evaluate the degrees of the extent of bone resection and fusion situation at final follow-up (Fig. 2). Pre-operative magnetic resonance imaging (MRI) showed normal nerve structure and no prominent variation in the planned osteotomy site.

\section{The surgical procedure.}

After informed consent was obtained, the patient was placed prone on the operating table and underwent general endotracheal anesthesia. Intraoperative neurophysiologic monitoring was performed throughout the procedure. The back was prepared and draped in the routine sterile fashion. The reference frame was attached to the spinous process of the T5 vertebra and surgical instruments were registered. The navigation probe located the right outer edge of the L2 facet joint on the skin surface (convex side). A longitudinal incision about $3 \mathrm{~cm}$ was made and the skin, subcutaneous tissue and deep fascia were sequentially cut off. An expandable tubular retractor was used with the retractor system fixed to the table using an articulating arm (Fig. 3,4). After blunt separation of muscle space with the index finger, sequentially larger tubular retractors were placed to allow a working portal.

Residual soft tissue at the base of the tube was removed to expose the pars, partial laminae and facet joint of the L2 vertebra (Fig. 3). The first step was to resect the inferior process of L1 and superior articular process of L2 using piezosurgery and osteotomes alternatively to try to preserve as much bone pieces as possible. Subsequently, then a partial removal of the laminae and removal of the ligamentum was performed to expose the L2 right nerve root and dura sac. The second step was to detach the transverse processes from the pedicle and then resect the pedicle stump on right side flush with the vertebral body as verified by the navigation probe. Third, the lateral vertebral cortex and the posterior wall of the vertebral body underneath the posterior vertebral cortex were resected as much as possible with piezosurgery and osteotomes alternatively (Fig. 4). The same step was performed on the left side, but the resection of the lamina and vertebral body of this side was less than that of the right side. A fixed tubular retractor was also used on this side. (Fig. 5). Once the posterior wall of the L2 vertebral body was thin enough, a substantial reverse-angled curette was placed between the anterior dura and the posterior vertebral cortex and subsequently pushed anteriorly to create a greenstick fracture of the posterior vertebral cortex. The fractured posterior cortex was then removed.

Percutaneously placed pedicle screws were inserted on both sides from T9 to L5 (except L2) assisted by navigation. On the left side, and a temporary rod was passed underneath the fascia and then secured to the connecting rod with the set screws (Fig. 6). The posterior elements (spinous process and ligaments) were completely resected using Leksell rongeurs and piezosurgery alternatively. The spinal canal was then enlarged centrally somewhat more with the use of a Kerrsion rongeur.

Contoured rods with a natural lumbar lordosis and an appropriate kyphosis at the thoracolumbar junction were passed freehand through all the extenders, and then sequentially reduced into the tulip of the screw 
head with the extenders. Significant compressive forces had to be applied to achieve proper osteotomy closure, which was confirmed by radiography and palpation while viewed through the tubular retractor. The rod was held in a strict sagittal plane as the reduction was achieved. The extenders were removed after the rod was locked in position with top locking screws. The wound was irrigated with saline. The facet joints were decorticated via osteotomy, and copious amounts of decorticated local bone autograft were then placed in each facet-pars complex. A sub-fascial drainage was placed through a separate stab incision and the wound was closed in layers.

\section{Post-operation procedure}

The patient began to ambulate three days after the operation and exercised in the stage of recovery. She was discharged from the hospital without complications and advised to come back to the hospital for a check at regular intervals. At the same time, anti-osteoporosis treatment was given.

\section{Results}

The duration of the operation was five hours and estimated blood loss was $600 \mathrm{ml}$. The patient received 2 units of packed red blood cells intraoperatively and was successfully extubated at the end of the procedure. There were no approach-related complications. VAS was 8.5 points at pre-operation and 2 points at final follow-up. The ODI score improved from 38 pre-operation to 9 at final follow-up. Maximal ambulation distance without pain increased from 500 feet to 5000 feet at final follow up.

Radiological measurement (Table. 1) and routine X-ray images and CT scans were obtained at pre- and post-operative follow up (Fig. 7,8). The wedging of the L2 vertebral body is clearly seen on the sagittal reconstructive film with complete union at the osteotomy site. No symptomatic proximal junction kyphosis was found on the lateral radiographic films. 
Table 1

Radiographic measures at each follow-up.

\begin{tabular}{|lllllll|}
\hline time & preoperation & 1week & 2 months & $\mathbf{8}$ months & 12months & 24months \\
\hline $\mathrm{TS}(\mathrm{mm})$ & 52 & 0 & 0 & 6 & 5 & 0 \\
\hline $\mathrm{Lcobb}\left({ }^{\circ}\right)$ & 40 & 12 & 14 & 14 & 14 & 14 \\
\hline $\mathrm{SVA}(\mathrm{mm})$ & 55 & 20 & 10 & 40 & 10 & 8 \\
\hline $\mathrm{T}_{11^{-}-\mathrm{L}_{3}}\left(^{\circ}\right)$ & 48 & 16 & 16 & 16 & 16 & 16 \\
\hline $\mathrm{TK}\left(^{\circ}\right)$ & 46 & 22 & 30 & 40 & 40 & 35 \\
\hline $\mathrm{LL}\left({ }^{\circ}\right)$ & 38 & 40 & 36 & 32 & 32 & 32 \\
\hline $\mathrm{PI}\left(^{\circ}\right)$ & 44 & 44 & 44 & 44 & 44 & 44 \\
\hline $\mathrm{PT}\left(^{\circ}\right)$ & 34 & 14 & 20 & 30 & 30 & 32 \\
\hline $\mathrm{SS}\left({ }^{\circ}\right)$ & 10 & 30 & 24 & 14 & 14 & 14 \\
\hline
\end{tabular}

\section{Discussion}

Adult spinal deformity is increasing in prevalence among older adults with comorbidities that increase the risks of surgery. MIS surgical techniques have been increasingly incorporated into the operative treatment of ASD, with a goal of less morbidity and tissue injury. Our case provides a promising method for surgically correcting kyphoscoliosis that needed posterior three column osteotomy in elderly patients. A PSO via tubular retractor system is not only correcting the severe rigid spinal deformity but also reducing high complication rates.

The selection of appropriate treatment for degenerative scoliosis is challenging because the condition is heterogeneous with diverse presentation of symptoms and variable results. Patients in whom conservative measures have failed are considered for surgical treatment. The use of MIS approaches for spinal fusion has become increasingly popular, leading some surgeons to explore its use for ASD treatment. Anand et al. [13] first described the use of several different MIS techniques to treat lumbar scoliosis in 2008. In that initial report, a combination of LLIF, axial lumbar interbody fusion, and percutaneous segmental instrumentation was used in 12 patients with a mean scoliosis of $18.9^{\circ}$. Although the magnitude of the scoliosis treated was mild, that study demonstrated the feasibility of an MIS approach to treat ASD. Mummaneni et al. [14] provided a framework for rational decision making for surgeons who are considering MIS versus open spine surgery based on the minimally invasive spinal deformity surgery (MISDEF) algorithm. Patients with mild flexible curves and primarily claudication/radicular symptoms are best treated with focal procedures (either decompressions and/or short segment fusions) that address individual symptom-driving segments as opposed to deformity correction. Patients with moderate deformities, the "Class 2" patients (LL-PI mismatch of 10-30 degrees, flexible major coronal curve $>20$ degrees that partially correct when supine, $S V A<6 \mathrm{~cm}$ ), are candidates 
for minimally invasive scoliosis correction using decompression and interbody fusion across the apex of the curvature to obtain correction along with percutaneous instrumentation. Patients with significant coronal and/or sagittal imbalance, the "Class 3" patients (ie, rigid deformities, iatrogenic flatback, etc.), are more likely to require a standard open approach with a three-column osteotomy for realignment. Park and colleagues [15] proposed a basic classification of MIS approaches for ASD that includes hybrid and circumferential MIS approaches. Both of the approaches resulted in similar and significant clinical improvement, as evidenced by decreased ODI and VAS pain scores. However, the rate of complications was higher with the hybrid approach than with the circumference MIS approach. However, MIS techniques have been reported to result in suboptimal sagittal plane correction or pseudarthrosis when used for severe deformities. [14]

How to perform minimally invasive three-column osteotomy for adult severe spinal deformity has always been a clinical problem. Wang and Bordon described the mini-open PSO $[16,17,18]$ in which posterior instrumentation is placed minimally invasive and only the osteotomy level and adjacent levels are exposed in an open fashion. The PSO was performed as in the standard open operation. Although this method was technically feasible in all cases with acceptable radiographic outcomes similar to open surgery, high complication rates associated with these deformity corrections remain problematic.

A tubular retractor system was first developed for microdiscectomy in 1999 by Foley and Smith [19]. Its basic concept is the foundation on which several contemporary approaches to minimally invasive posterior lumbar fusion are based. The system consists of a series of concentric dilators and thin-walled tubular retractors of variable length. The spine is accessed via serial dilation of the natural cleavage plane between muscle fascicles, instead of a more traumatic muscle-stripping approach. All of the midline supporting musculoligamentous structures are left intact with this technique. An appropriated sized working channel is created that permits spinal decompression and fusion. The decreased soft tissue disruption in the MIS technique has been associated with significantly decreased blood loss, shorter length of hospitalization, and an expedited return to work. The tubular retractor approach can be utilized for minimally invasive lumbar fusion via posterolateral onlay, PLIF or TLIF.

In this rigid adult spinal deformity case, coronal imbalance (C7-CSVL distance : $5.2 \mathrm{~cm}$, coronal Cobb angle : 40 degrees) and sagittal imbalance (SVA $5.5 \mathrm{~cm}$ ) was significantly manifested in the radiographic films. According to MISDEF algorithm, this case is suitable for a standard open approach with three column osteotomy for realignment. However, the patient and her family members refused to perform open surgery because of concerns about the complications. In recent years, we have accumulated a lot of experience from minimally invasive surgery via tubular retractor and traditional PSO. By careful assessment of the physical and mental state and communication with family members, we decided on performing MIS PSO. This was approved by Hospital Ethics Committees.

After performing this challenging surgery successfully, we reviewed articles and found only a few papers focusing on minimally invasive PSO via tubular retractor. In 2008, Voyadzis et al [20] performed nine PSOs using a minimally invasive approach in six cadavers via bilateral $X$ tubes ((Medtronic Sofamor 
Danek)) or Quadrant retractor system. Through the working channels, a complete wide laminectomy was performed by two surgeons simultaneously, then the pedicle and posterior and lateral walls of the vertebral body were removed with a high-speed drill, Kerrison rongeur, and osteotomies with fluoroscopic assistance. Complete resection of the posterior body wall was confirmed with instrumented palpation and visualization through both working channels. Compression and osteotomy closure were then accomplished with fluoroscopic assistance after percutaneous pedicle screw placement. A mean Cobb angle change of 15 degrees after lumbar PSO closure was obtained. Nearly complete dorsal element resection was confirmed with postprocedural CT scans. Inspection of the thecal sac after PSO closure revealed no impingement from dorsal structures. There were no cases of dura violation or obvious injury to the neural elements. They also performed minimally invasive posterior vertebrectomy via tubular retractor in the six cadavers and two clinical cases for a T6 burst fracture and a T4-T5 plasmacytoma with satisfactory decompression and reconstruction.

Compared with Voyadzis' PSO in cadavers [20], our surgery was the first case with rigid spinal deformity to perform PSO via tubular retractor in the literature. The clinical results were satisfactory and coronal and sagittal imbalance was corrected. The global kyphosis and lumbar Cobb angle were 16 degrees and 14 degrees at final follow-up; a correction of nearly 30 degrees, with the amount of correction similar to open PSO. With the advance of techniques such as navigation systems, intraoperative spinal cord monitoring is the necessary condition for a guarantee of surgeries safety [21]. A sagittal deformity that is combined with coronal imbalance is better treated with an asymmetric pedicle subtraction osteotomy so that the coronal deformity is corrected rather than exacerbated as described in the article. Although only one patient was included in our paper, we believe in the awareness on operation beyond the technique rather than on operation itself. Our method provided an effective and successfully approach to solve this adult deformity that required us to perform PSO.

As with any novel surgical technique, a learning curve is associated with the development of proficient technical skills. The first requirement is that the surgeon must be familiar with three-dimensional spinal anatomy within the limited field of view without exposing structures that are not being surgically treated. The second is that the surgeon must become facile working through a significantly smaller exposure using instruments that are longer and are frequently bayoneted. The third and the most important is the selection of a suitable case. The indications of MIS PSO are similar to those of open surgery, but MIS PSO is also likely to be more technically demanding than are corresponding open procedures.

\section{Conclusion}

Minimally invasive technology is widely applied in spinal surgeries and has a far-reaching influence in medical decision making. MIS PSO is a feasible option for the treatment of certain subtypes of adult spinal deformity. However, not every adult patient with deformity can be managed with MIS PSO. Proper patient selection is the key to successfully treating adult patients with deformity using MIS PSO.

\section{Abbreviations}


ASD: adult spinal deformity; PSO: pedicle subtraction osteotomy; MIS: minimally invasive surgery; MIS PSO: minimally invasive pedicle subtraction osteotomy; VAS: visual analog scale; PA: posterior-anterior; CSVL: center of the sacrum; TK: thoracic kyphosis; LL: lumbar lordosis; GK: global kyphosis; SS: sacral slope; PT: pelvic tilt; PI: pelvic incidence; SVA: sagittal vertical axis; CT: computed tomography.

\section{Declarations}

\section{Ethics approval and consent to participate}

This study was approved by the Ethics Committee of Shanxi Bethune Hospital (YXLL-2019-136). The patient provided written informed consent prior to the study.

\section{Consent for publication}

All authors provide their consent to the subscription and publication of this manuscript and all related files.

\section{Availability of data and materials}

The raw data were collected and analyzed by the authors and are available upon reasonable request from the corresponding author.

\section{Competing interests}

The authors declare that they have no competing interests.

\section{Funding}

Not applicable.

\section{Authors' contributions}

Zejun Xing wrote the initial draft, did the review of the literature, and edited the manuscript. Zhuo Ma and Ma Xun reviewed and edited the manuscript. Xun Ma did the surgery, provided intraoperative images, and edited the manuscript.

\section{Acknowledgements}

Not applicable.

\section{References}

1. Schwab F, Dubey A, Gamez L, et al. Adult scoliosis: prevalence, SF-36, and nutritional parameters in an elderly volunteer population. Spine (Phila Pa 1976) 2005; 30(9):1082-85. 
2. Smith J S, Shaffrey C I, Fu K M, et al. Clinical and radiographic evaluation of the adult spinal deformity patient. Neurosurg Clin N Am2013; 24(2):143-156.

3. Bridwell KH, Lewis SJ, Lenke LG, et al. Pedicle subtraction osteotomy for the treatment of fixed sagittal imbalance. J Bone Joint Surg Am 2003; 85-A(3):454-63.

4. Kim YJ, Bridwell KH, Lenke LG, et al. Results of lumbar pedicle subtraction osteotomies for fixed sagittal imbalance: a minimum 5-year follow-up study. Spine (Phila Pa 1976) 2007; 32(20):2189-97.

5. Thomasen E. Vertebral osteotomy for correction of kyphosis in ankylosing spondylitis. Clin Orthop Relat Res 1985 Apr; (194) :142-52.

6. Gill JB, Levin A, Burd T, Longley M. Corrective osteotomies in spine surgery. J Bone Joint Surg Am2008; 90:2509-20. DOI: 10.2106/JBJS.H.00081.

7. Deviren V, Scheer JK, Ames CP. Technique of cervicothoracic junction pedicle subtraction osteotomy for cervical sagittal imbalance: report of 11 cases. J Neurosurg Spine 2011;15:174-81. DOI: 10.3171/2011.3.SPINE10536.

8. Hyun SJ, Kim YJ, Rhim SC. Spinal pedicle subtraction osteotomy for fixed sagittal imbalance patients. World J Clin Cases 2013;1:242-8. DOI: 10.12998/wjcc.v1.i8.242.

9. Buchowski J M, Bridwell K H, Lenke L G, et al. Neurologic complications of lumbar pedicle subtraction osteotomy: a 10-year assessment. Spine (Phila Pa 1976) 2007 ; 32(20):2245-52.

10. Kim CW. Scientific basis of minimally invasive spine surgery: prevention of multifidus muscle injury during posterior lumbar surgery. Spine (Phila Pa 1976) 2010;35(26 Suppl):S281-6. DOI: 10.1097/BRS.0b013e3182022d32.

11. Parker SL, Adogwa O,Witham TF, Aaronson OS, Cheng J,McGirt MJ. Post-operative infection after minimally invasive versus open transforaminal lumbar interbody fusion (TLIF): literature review and cost analysis. Minim Invasive Neurosurg 2011;54(1):33-7. DOI: 10.1055/s-0030-1269904. Epub 2011 Apr 19.

12. McGirt MJ, Parker SL, Lerner J, Engelhart L, Knight T,Wang MY. Comparative analysis of perioperative surgical site infection after minimally invasive versus open posterior/transforaminal lumbar interbody fusion: analysis of hospital billing and discharge data from 5170 patients. J Neurosurg Spine 2011;14(6):771-8. DOI: 10.3171/2011.1.SPINE10571. Epub 2011 Mar 18.

13. Anand N, Baron EM, Thaiyananthan G, Khalsa K, Goldstein TB. Minimally invasive multilevel percutaneous correction and fusion for adult lumbar degenerative scoliosis: a technique and feasibility study. J Spinal Disord Tech 2008;21(7):459-67. DOI: 10.1097/BSD.0b013e318167b06b.

14. Mummaneni $P$ V, Shaffrey $C$ I, Lenke $L G$, et al. The minimally invasive spinal deformity surgery algorithm: a reproducible rational framework for decision making in minimally invasive spinal deformity surgery. [J]. Neurosurgical Focus 2014;36(5): E6. DOI: 10.3171/2014.3.FOCUS1413.

15. Park P,Wang MY, Lafage $V$, et al. Comparison of two minimally invasive surgery strategies to treat adult spinal deformity. J Neurosurg Spine. 2015;22(4):374-380.

16. Wang MY, Bordon G. Mini-open pedicle subtraction osteotomy as a treatment for severe adult spinal deformities: case series with initial clinical and radiographic outcomes. J Neurosurg Spine 
2016;24(5):769-76. DOI: 10.3171/2014.9.SPINE131004. Epub 2015 Jan 30.

17. Wang M Y. Miniopen pedicle subtraction osteotomy: surgical technique and initial results. Neurosurg Clin N Am 2014; 25(2):347-51. DOI: 10.1016/j.nec.2013.12.012.

18. Chou, Dean, Lau, et al. The mini-open pedicle subtraction osteotomy for flat-back syndrome and kyphosis correction: operative technique. Oper Neurosurg (Hagerstown) 2016 Dec 1;12(4):309-316. DOI: 10.1227/NEU.0000000000001167

19. Foley KT, Smith MM®Rampersaud YR. Microendoscopic approach to far-lateral lumbar disc herniation. Neurosurg Focus. 1999;7(5):e5.DOI: 10.3171/foc.1999.7.6.6.

20. Jean-Marc V, Gala VC, O’Toole JE,et al. Minimally invasive posterior osteotomies. Neurosurgery.2008;63(3 Suppl):204-10. DOI: 10.1227/01.NEU.0000320430.37577.B7.

21. Jarvis J G, Strantzas S, Lipkus M, et al. Responding to neuromonitoring changes in 3-column posterior spinal osteotomies for rigid pediatric spinal deformities. Spine (Phila Pa 1976) 2013 Apr 15;38(8):E493-503. DOI: 10.1097/BRS.0b013e3182880378.

\section{Figures}



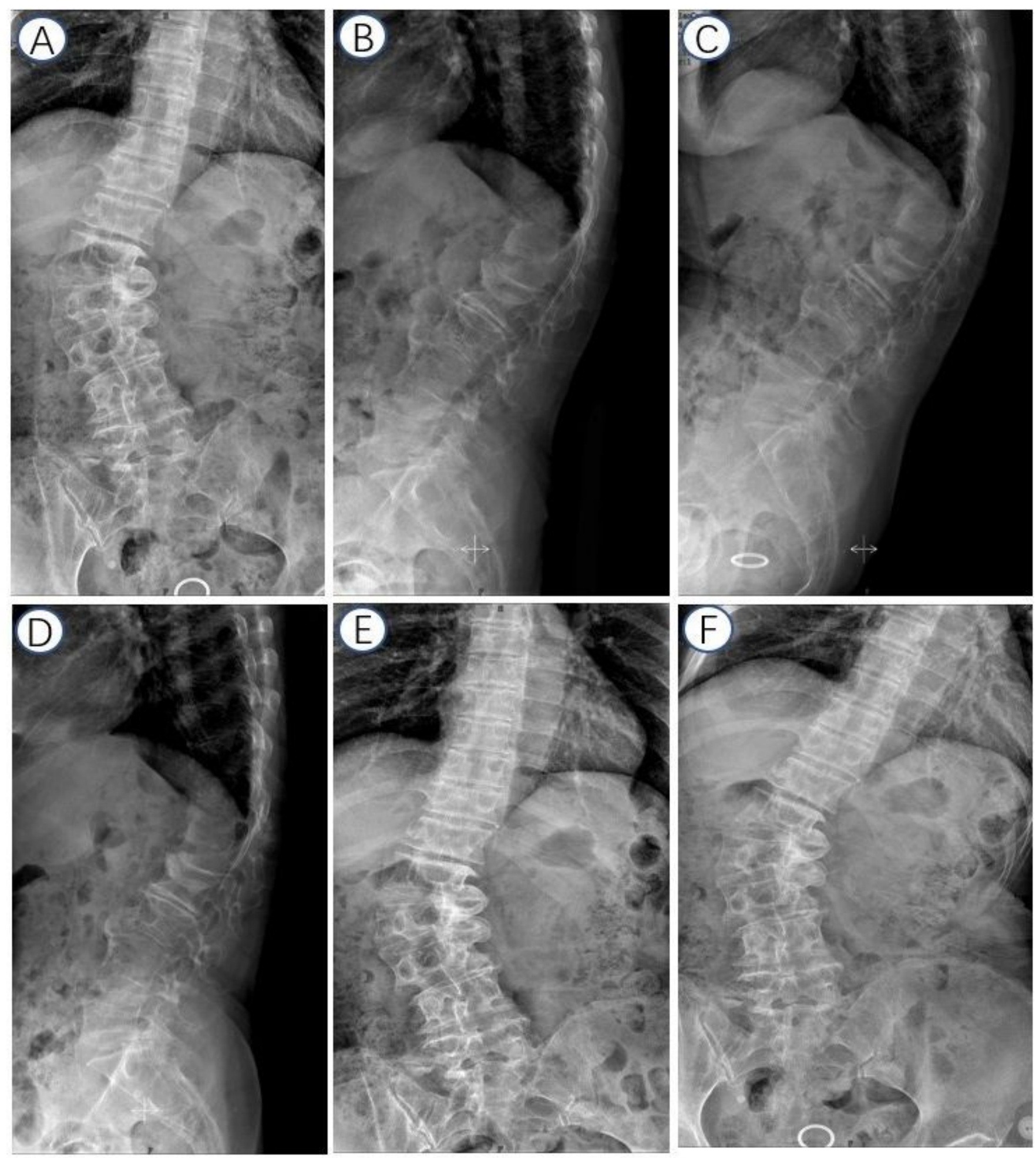

\section{Figure 1}

A. Preoperative A-P X-ray showed lumbar curve (T12-L4) Cobb is 40 degrees. B. Lateral X-ray showed global kyphosis (T11-L3) is 48 degrees. C -F. Lateral views in flexion and extension and supine bending views showed rigid kyphoscoliosis deformity. 

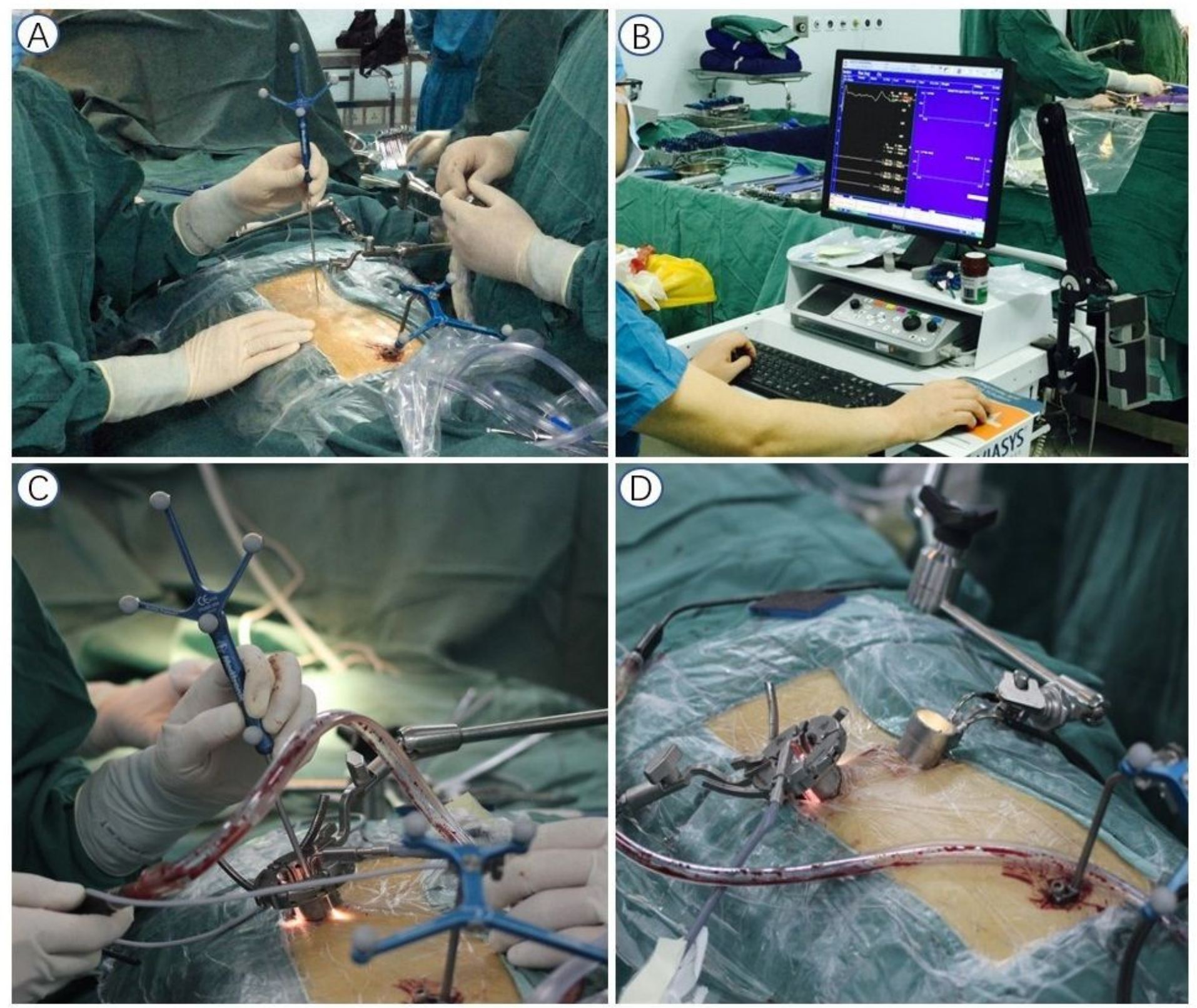

Figure 2

Intraoperative views. A. Installation of navigation reference frame. B. Sensory and motor evoked potential (SEP and MEP) monitoring. C. Installing right tubular retractor. D. Installing left tubular retractor. 

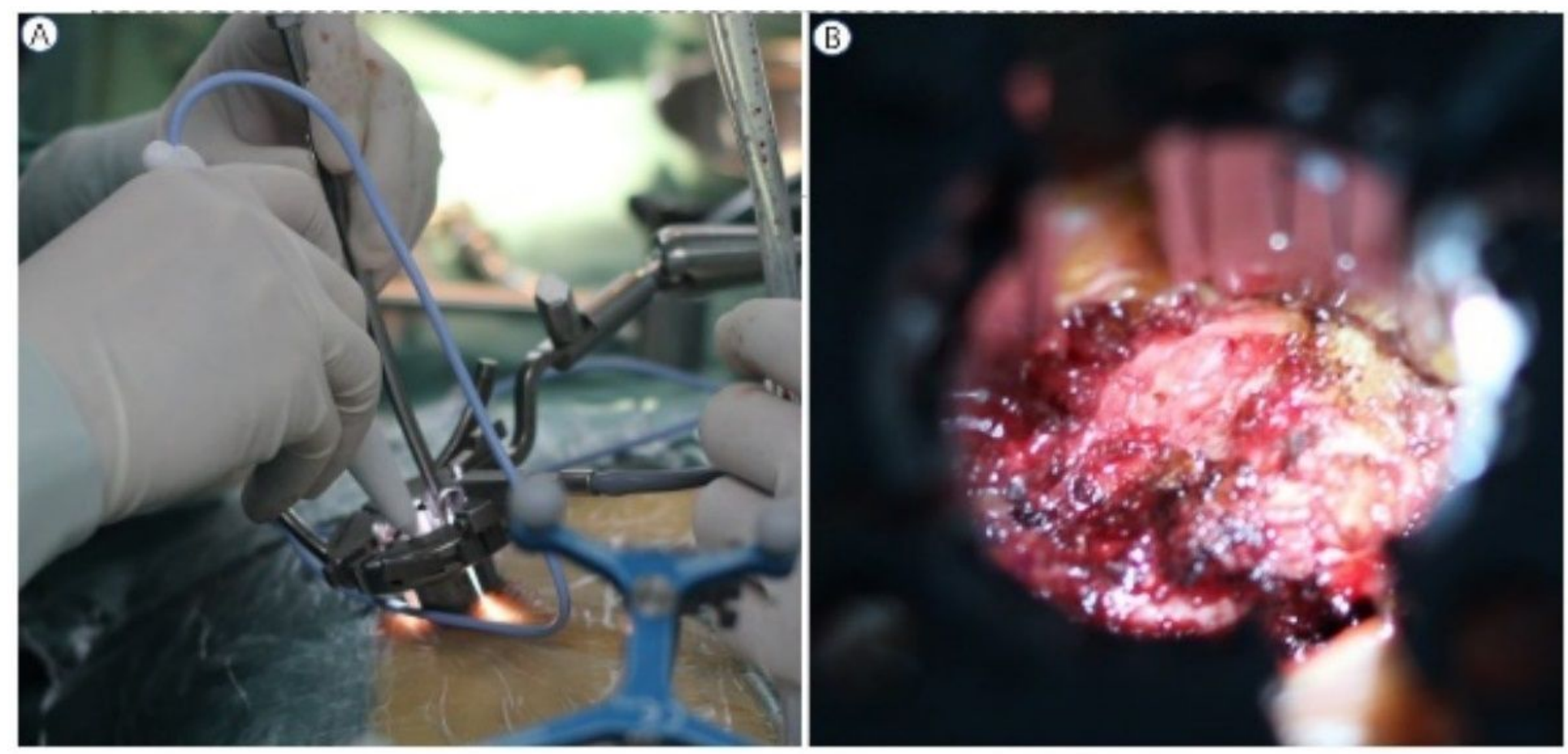

\section{Figure 3}

Intraoperative views. A. remove the residual soft tissue and muscles utilizing electrocautery and pituitary rongeur. B. Tubular retractor image demonstrating the visualization of lamina, facet joint, and transverse processes of L2 vertebra.
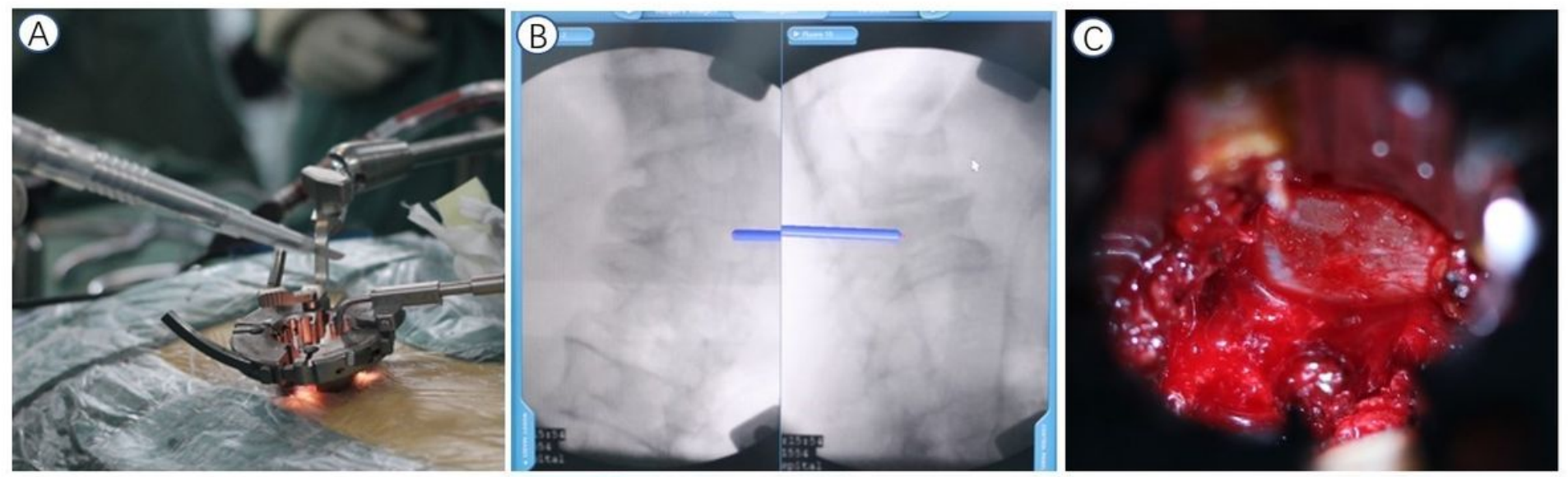

(D)
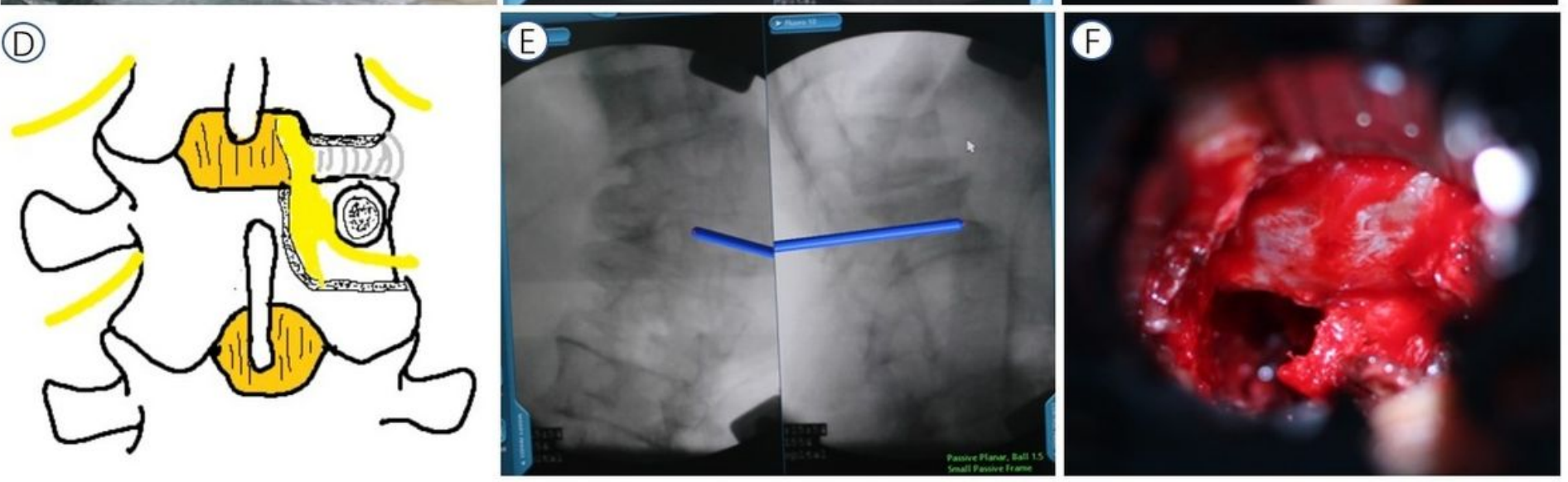


\section{Figure 4}

Intraoperative osteotomy of the right side. A. intraoperative piezosurgery. B. Navigation probe to identify the spinal structure via working channel. C. Tubular retractor image demonstrating the visualization of the dura sac and nerve root after resecting the lamina and articular process. D. Simplified diagram of the right side PSO. E. Navigation probe to measure the osteotomy depth. F. Tubular retractor image demonstrating interosteotomy space.
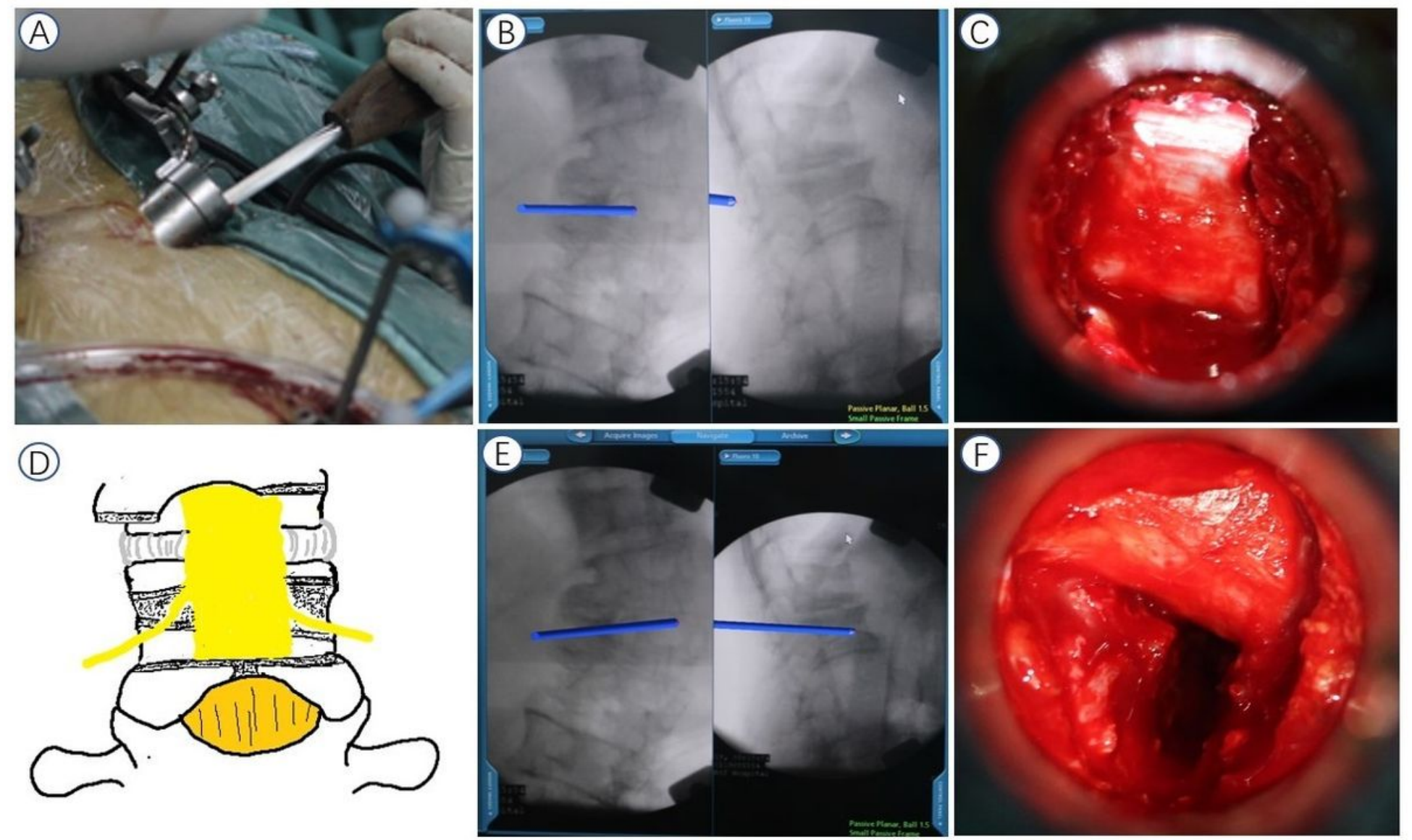

Figure 5

Intraoperative osteotomy of left side. A. intraoperative osteotomes. B. Navigation probe to identify the spinal structure via working channel. C. Tubular retractor image demonstrating the visualization of the dura sac and nerve root after resecting the lamina and articular process. D. Simplified diagram of the left side PSO. E. Navigation probe to measure the osteotomy depth. F. Tubular retractor image demonstrating inter-osteotomy space. 

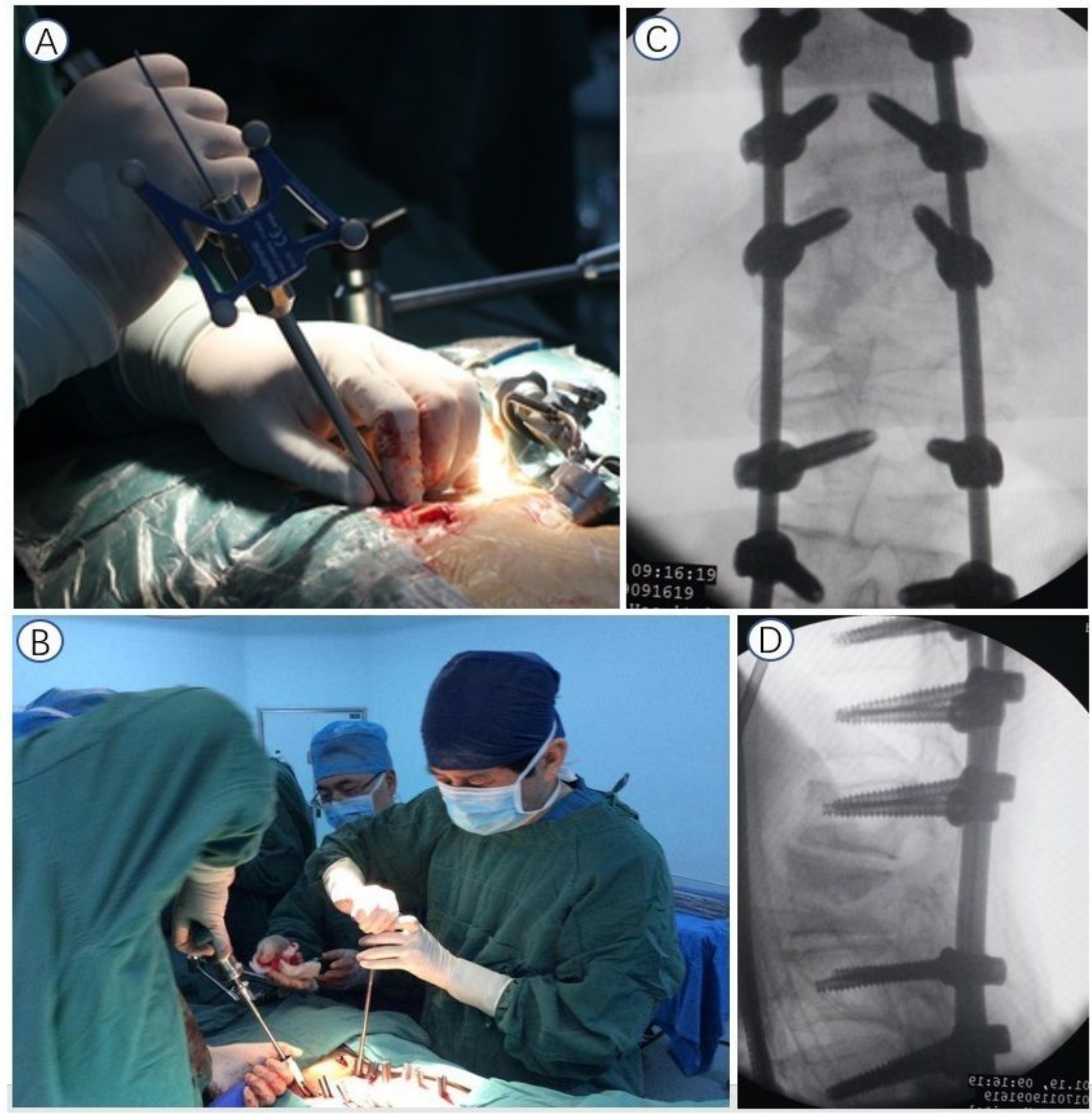

\section{Figure 6}

Percutaneous pedicle screw placement from T9 L5 was performed (A-B). Final osteotomy site was closed and the curve correction was achieved using fluoroscopy during the operation (C-D). 


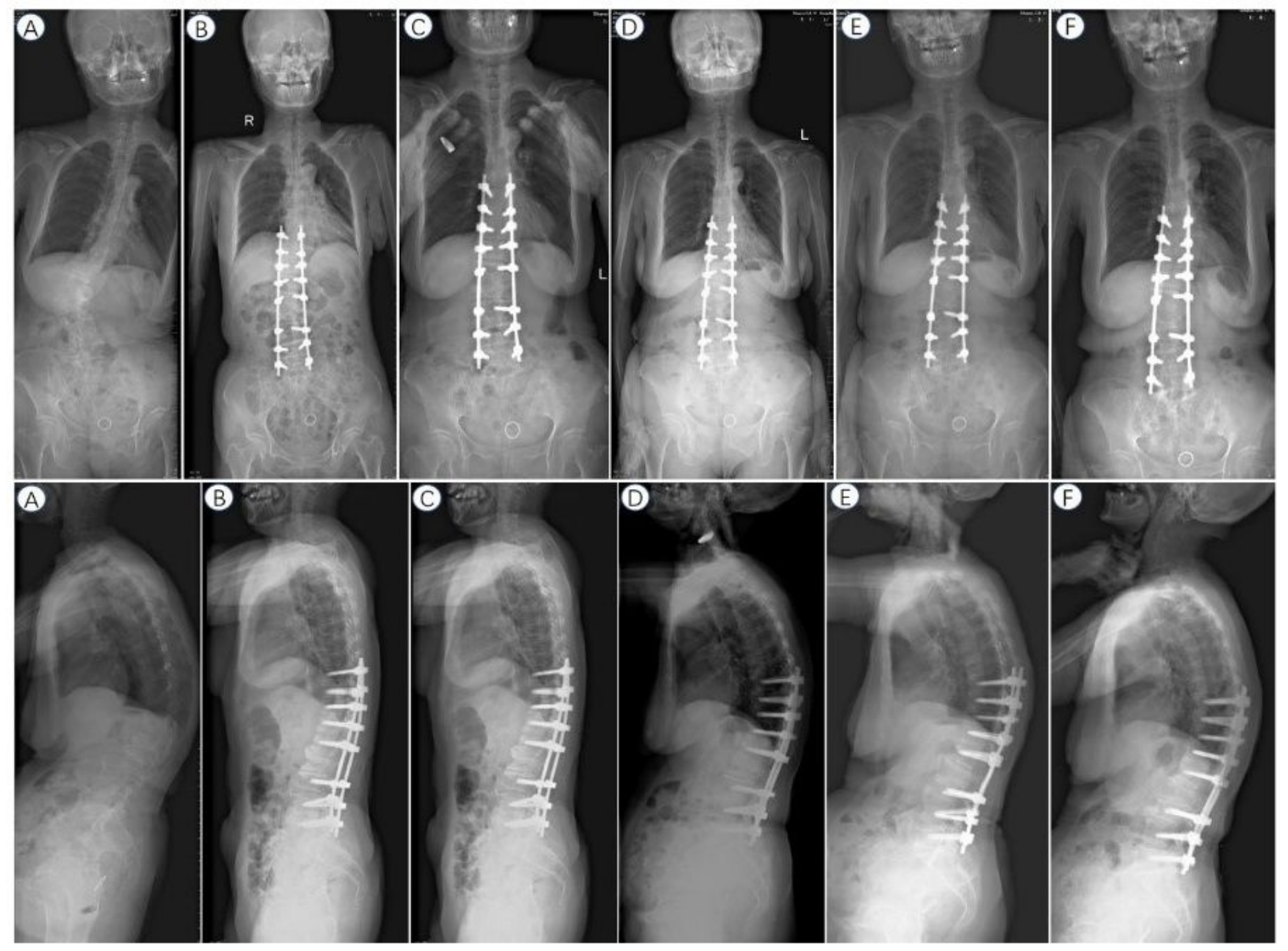

\section{Figure 7}

The standing 36-inch posteroanterior (PA) and lateral views pre-operation and all follow-up after operation. A. pre-operation. B. 1 week after operation. C. 2 months after operation. D. 8 months after operation. E. 12 months after operation. F. 24 months after operation.

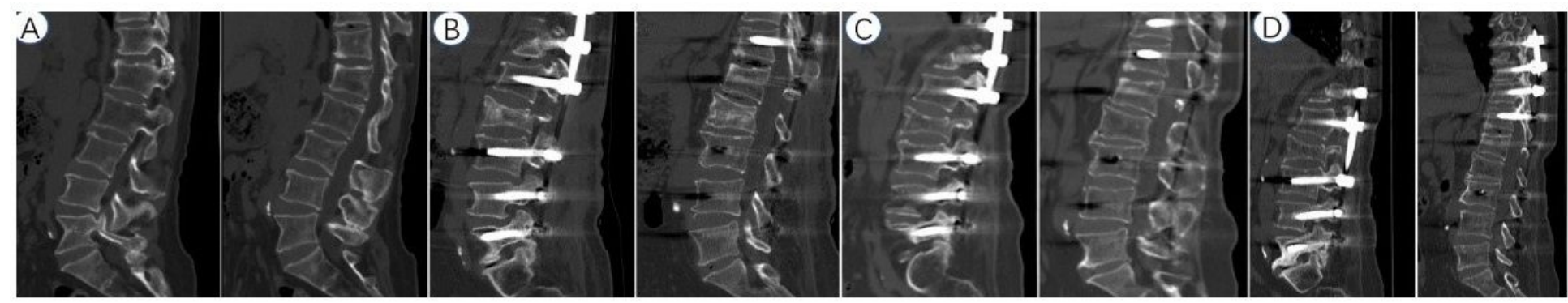

\section{Figure 8}

CT sagittal reconstruction views. A. Pre-operation. B. Osteotomy line is clear at 2 months after operation. C. Wedge-shaped change of L2 after 12 months follow-up. D. Wedge-shaped change of L2 after 24 
months follow-up.

Page 18/18 